\title{
Coil profile estimation strategies for parallel imaging with hyperpolarized 13C MRI
}

Hansen, Rie Beck; Sánchez-Heredia, Juan Diego; Bøgh, Nikolaj; Hansen, Esben Søvsø Szocska; Laustsen, Christoffer; Hanson, Lars G. ; Ardenkjær-Larsen, Jan Henrik

\section{Published in:}

Magnetic Resonance in Medicine

Link to article, DOI:

$10.1002 / \mathrm{mrm} .27892$

Publication date:

2019

Document Version

Peer reviewed version

Link back to DTU Orbit

Citation (APA):

Hansen, R. B., Sánchez-Heredia, J. D., Bøgh, N., Hansen, E. S. S., Laustsen, C., Hanson, L. G., \& ArdenkjærLarsen, J. H. (2019). Coil profile estimation strategies for parallel imaging with hyperpolarized $13 \mathrm{C}$ MRI.

Magnetic Resonance in Medicine, 82(6), 2104-2117. https://doi.org/10.1002/mrm.27892

\section{General rights}

Copyright and moral rights for the publications made accessible in the public portal are retained by the authors and/or other copyright owners and it is a condition of accessing publications that users recognise and abide by the legal requirements associated with these rights.

- Users may download and print one copy of any publication from the public portal for the purpose of private study or research.

- You may not further distribute the material or use it for any profit-making activity or commercial gain

- You may freely distribute the URL identifying the publication in the public portal 


\section{Coil Profile Estimation Strategies for Parallel IMAGING WITH HYPERPOLARIZED ${ }^{13} \mathrm{C}$ MRI}

Author list: Rie B Hansen ${ }^{1}$, Juan Diego Sánchez-Heredia ${ }^{1}$, Nikolaj Bøgh ${ }^{2}$, Esben Søvsø Szocska Hansen ${ }^{2}$, Christoffer Laustsen ${ }^{2}$, Lars G Hanson ${ }^{1,3}$, Jan H Ardenkjær-Larsen ${ }^{1}$

Affiliations: ${ }^{1}$ Department of Health Technology, Technical University of Denmark, Kgs. Lyngby, Denmark; ${ }^{2}$ MR Research Centre, Aarhus University, Aarhus, Denmark; ${ }^{3}$ Danish Research Centre for Magnetic Resonance, Centre for Functional and Diagnostic Imaging and Research, Copenhagen University Hospital Hvidovre, Denmark

Correspondence to:

Jan Henrik Ardenkjær-Larsen

Technical University of Denmark

Department of Health Technology

Ørsted Plads, bldg 349, office 126

DK - 2800 Kgs. Lyngby

E-mail: jhar@dtu.dk

Word count: 5101 (body of the text) 


\section{ABSTRACT}

Purpose: To investigate auto- and pre-calibration coil profile estimation for parallel imaging reconstruction of hyperpolarized ${ }^{13} \mathrm{C}$ MRI volumetric data.

Methods: Parallel imaging reconstruction was studied with three different approaches for coil profile estimation: auto-calibration, phantom calibration and theoretical calibration. Acquisition was performed with a 3D stack-of-spirals sequence with spectral-spatial excitation and Cartesian undersampling. Parallel imaging reconstructions were done with conjugate gradient SENSE and 3D gridding with inhomogeneity correction. The approaches were compared in simulations with different SNR, through phantom experiments, and in an in vivo pig study focused on the kidneys. All imaging was done with a rigid home-built 12 -channel ${ }^{13} \mathrm{C}$ receive coil at $3 \mathrm{~T}$.

Results: The phantom calibrated and theoretical approaches resulted in the best structural similarities in simulations, and demonstrated higher image quality in the phantom experiments compared to the auto-calibrated approach. In vivo mapping of pyruvate uptake and lactate conversion improved for accelerated acquisitions due to a better temporal resolution. From a practical and image quality point of view, use of theoretical coil profiles led to improved results compared to the other approaches.

Conclusion: The success of the theoretical coil profile estimation demonstrates a negligible effect of load on sensitivity profiles at the carbon frequency at $3 \mathrm{~T}$. Through theoretical or phantom calibrated parallel imaging, accelerated 3D volumes could be reconstructed with sufficient sensitivity, temporal and spatial resolution to map the metabolism of kidneys exemplifying abdominal organs. This approach overcomes a critical step in the clinical translation of parallel imaging in hyperpolarized ${ }^{13} \mathrm{C}$ MR.

Keywords: ${ }^{13} \mathrm{C}$ MRI; Hyperpolarization; Parallel Imaging; RF Coil Array; Metabolic imaging; Coil sensitivity estimation 


\section{INTRODUCTION}

Hyperpolarized ${ }^{13} \mathrm{C}$ MR offers unique opportunities for measuring first pass perfusion and fast metabolic processes in vivo, e.g., in tumors $(1,2)$ or the heart $(3,4)$. However, the short-lived nature of the substances' magnetization caused by $T_{1}$ relaxation, excitation depletion and metabolism places high demands on acquisition time. In order to prevent signal loss while acquiring large volumetric field-of-views (FOVs) with high spatial resolution, and in order to fully capture substrate uptake and rapid metabolic conversion, fast data acquisition is crucial.

Parallel imaging is a method that uses spatial information inherent to each coil element of multichannel coils to reduce scan times (5). Conventional parallel imaging results in an SNR reduction given by the square-root of the acceleration factor multiplied with the geometry factor (g-factor). However, for parallel imaging with hyperpolarized ${ }^{13} \mathrm{C} M R I$, the inherent SNR is only reduced by the g-factor, which may be countered by reduced relaxation losses (6). Increased encoding efficiency allows higher temporal resolution or a larger FOV in hyperpolarized imaging.

The majority of parallel imaging methods requires estimation of the coil sensitivity profiles either explicitly as for SENSE (7) or implicitly as in GRAPPA kernel estimation (8). Explicit estimation of coil sensitivities is normally done using either a separate calibration scan (pre-calibration) or low-resolution scans acquired by measuring the central part of k-space with full Nyquist sampling (auto-calibration) while undersampling the outer parts of k-space. Self-calibrating parallel imaging methods exist as well. One such method is SAKE (9-11), which does not explicitly involve coil sensitivity estimation, but instead uses priors in the multi-channel dataset to formulate the reconstruction as a low-rank matrix completion problem. However, SAKE requires non-uniform undersampling to be able to resolve aliasing artifacts, which limits its general applicability. Traditional parallel imaging methods, such as SENSE, on the other hand, are applicable to all available pulse sequences (5). If accurate sensitivity maps are available, SENSE is expected to work best (8).

Estimating coil sensitivity profiles in the context of hyperpolarized ${ }^{13} \mathrm{C}$ MRI poses new challenges. Acquiring reference data during the subject pre-scan, as for conventional proton parallel imaging, is not feasible, due to the low natural abundance of ${ }^{13} \mathrm{C}$. Instead, the reference data will either need to be acquired as an integral part of the acquisition through auto-calibration $(6,12,13)$, or need to be estimated accurately from a phantom measurement (14) or through theoretical calculations (15) in a pre-calibration step. The first choice limits the maximum achievable acceleration rate, especially in the context of the comparatively small matrix sizes 
used in hyperpolarized ${ }^{13} \mathrm{C}$ acquisitions, and the latter choices might prove sub-optimal for flexible arrays and in situations where the subject significantly influences the sensitivity profiles. However, this may offer a particular opportunity for ${ }^{13} \mathrm{C}$ imaging due to the lower Larmor frequency. With a resonance frequency of $32.13 \mathrm{MHz}$ at $3 \mathrm{~T}$, the geometry of the RF fields are largely independent of the imaged object; i.e. minimally affected by sample loading (16).

The objective of this study is to determine the optimal strategy for estimating reliable coil sensitivity profiles for parallel imaging reconstruction of hyperpolarized ${ }^{13} \mathrm{C}$ MR data using SENSE. Using a rigid home-built multi-channel coil, we perform simulations under different SNR conditions, phantom experiments, and evaluation in an in vivo pig study. A major advantage of a fixed coil geometry is the possibility of using simulated coil profiles for reconstruction, which is explored here.

Three different approaches to sensitivity calibration are compared:

1. Auto-calibration; estimating sensitivity maps from a fully sampled k-space center.

2. Phantom pre-calibration; estimating sensitivity maps from a thermally polarized phantom prior to placing the subject in the scanner.

3. Theoretical pre-calibration; estimating sensitivity maps based on Biot-Savart integration with respect to the geometric localization of the coil-array relative to the subject.

As exact hardware-induced phase delays and receiver amplifier gains can be difficult to predict theoretically, approach 3 will be calibrated based on phantom data.

The three approaches are tested with a 3D stack-of-spirals parallel imaging sequence accelerated in the stacked dimension and with spectral-spatial (SPSP) excitation. The sequence was designed with focus on abdominal ${ }^{13} \mathrm{C}$ imaging as this especially will benefit from parallel imaging, when covering large FOVs.

\section{METHODS}

The same 3D stack-of-spirals sequences were used in simulations, in phantom and in vivo. A

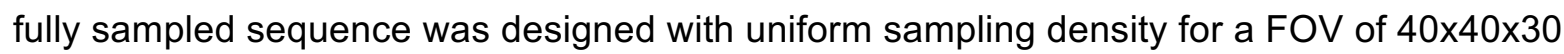
$\mathrm{cm}^{3}$, a nominal in-plane resolution of $5 \mathrm{~mm}$, and through-plane resolution of $10 \mathrm{~mm}$ (reconstructed matrix size $80 \times 80 \times 30$ ). Assuming a $\mathrm{T}_{2}{ }^{*}$ relaxation time of $20-25 \mathrm{~ms}$ (for lactate with $\mathrm{J}_{\mathrm{CH}}$-dominated signal decay) (17), the spiral readout duration was chosen to be $45 \mathrm{~ms}$ (18). Consequently, by applying a $15-\mathrm{Hz}$ Gaussian line-broadening filter to each spiral k-space 
readout, the actual resolution becomes $1 \mathrm{~cm}$ (isotropic). The pulse sequence including SPSP excitation is shown in Figure 1a. The expected spectral and spatial selectivity of the used SPSP pulse are shown in Figure 1b. The pulse profile was simulated using tools from the "SpectralSpatial RF Pulse Design MATLAB package" (19).

Two undersampled versions of the sequence were designed, both with an acceleration rate $\mathrm{R}=$ 2: 1) a uniformly undersampled stack-of-spirals, skipping every second phase-encode in the stacked dimension, 2) an undersampled stack-of-spirals with an undersampling factor of 3 in the outer parts of k-space and a fully sampled center of k-space (auto-calibration scheme). See Figure 1c.

All data were acquired on an MR750 3T scanner (GE Healthcare, Waukesha, WI, USA) with a custom-made coil setup consisting of a linear ${ }^{13} \mathrm{C}$ volume coil for transmission, and a 12element array for reception (see Figure 2). The transmit coil is of the clamshell type $(14,20)$ with a diameter of $400 \mathrm{~mm}$, and two opposed loops covering $120^{\circ}$ in order to resemble the current pattern of a saddle coil and hereby maximize field homogeneity (21). The receive array consists of two rows of six coils each mounted over a cylinder with a diameter of $300 \mathrm{~mm}$. The individual coil diameter is $130 \mathrm{~mm}$. The array was designed following the decoupling strategy described in (22), allowing for a non-overlapped geometry in order to minimize the g-factor penalty. The measured $Q_{\text {unloaded }} / Q_{\text {loaded }}$ ratio was $580 / 120$. Both coils are mounted over fiberglass cylinders to provide a high mechanical stability and to keep a stable relative position between elements and transmit-receive coils. All ${ }^{1} \mathrm{H}$ images were acquired using the body coil of the scanner.

For the phantom experiments a large volume ethylene glycol phantom was used (natural abundance ${ }^{13} \mathrm{C}$; triplet with $\mathrm{J}_{\mathrm{CH}}$ of $\sim 142 \mathrm{~Hz}$ ). The phantom is cylindrical with a $25-\mathrm{cm}$ outer diameter and a length of $20 \mathrm{~cm}$. The phantom was loaded with $\mathrm{NaCl}(17 \mathrm{~g} / \mathrm{L})$, which resulted in a conductivity close to that of muscle tissue at $32.13 \mathrm{MHz}(\sim 0.66 \mathrm{~S} / \mathrm{m})$. The $\mathrm{T}_{1}$ relaxation time constant of the phantom was measured to be $\sim 0.7 \mathrm{~s}$.

\section{SIMULATIONS}

All sequence simulations were performed in the flexible MR simulator JEMRIS (23) and analyzed using MATLAB (R2016a, MathWorks, Natick, MA, USA). The 3D stack-of-spirals sequences were designed with uniform sampling density using MATLAB scripts from the Multinuclear Spectroscopy (MNS) research pack (GE Global Research, Munich, Germany) as starting point. The sensitivity profiles of the 12-element receive array were simulated using the electromagnetic simulation suite CST (Darmstad, Germany). The simulation accounted for the 
electronic losses of the components and the decoupling obtained from mismatched preamplifiers (using circuit co-simulation). The preamplifiers were assumed noise-free and therefore not contributing to noise correlation. Both sequence and receive array profiles were imported into JEMRIS together with a digital phantom to imitate the phantom study. The transmit coil profile was assumed uniform. The digital phantom was designed as a replica of the physical phantom and simulated with an 8-degree tilt along the z-axis (see Supporting Information Figure S1). The following relaxation parameters were used in the simulation: $\mathrm{T}_{1}=$ $1000 \mathrm{~ms} ; \mathrm{T}_{2}=200 \mathrm{~ms}$; and $\mathrm{T}_{2}{ }^{*}=50 \mathrm{~ms}$ (simulating $\mathrm{T}_{2}{ }^{*}$ properties of pyruvate) (24). The simulation was performed with a $\pm 15 \mathrm{~Hz}$ macroscopic off-resonance distribution and hard pulse singlet excitation. Off-resonance effects of the SPSP excitation were therefore not simulated. Different levels of complex Gaussian noise were added to test reconstruction fidelity.

The MATLAB implementation of the structural similarity index measure (SSIM) (25) was used to assess image quality of the simulation results compared to the true simulated sample (the digital phantom). In the following, when a SSIM value is stated, this represents the mean value within the object mask.

\section{PHANTOM PRE-CALIBRATION}

Phantom pre-calibration sensitivity maps were generated based on a multi-slice CSI acquisition. The sequence was setup to cover the full phantom $\left(F O V=30 \times 30 \times 21 \mathrm{~cm}^{3}\right)$ with an isotropic resolution of $1.5 \mathrm{~cm}$. TR $=500 \mathrm{~ms}$; flip angle $=60^{\circ} ; 8$ averages. Total acquisition time of $6 \mathrm{~h} 13$ $\mathrm{min}$. To enable registration of the pre-calibrated coil profiles, three coil markers $(5 \mathrm{~mm}$ diameter oval vitamin E capsules) were placed in the center of three different coil loops of the receive array.

\section{PHANTOM EXPERIMENTS}

Phantom experiments were performed with the fully sampled 3D stack-of-spirals with: $T R=1 \mathrm{~s}$; flip angle $70^{\circ}$; SPSP pulse with fly-back gradients (22.44 ms); 60 vs. 120 averages. The fully sampled stack-of-spirals data were reconstructed based on 60 averages, whereas retrospectively undersampled datasets for the two undersampling schemes were created and reconstructed based on 120 averages to give identical total signal acquisition time.

Main field $\left(B_{0}\right)$ inhomogeneity was estimated using the dual-echo method (26), to enable inhomogeneity correction in the post-processing. The $\mathrm{B}_{0}$-field was mapped at the proton frequency and subsequently scaled with respect to the ratio of the ${ }^{13} \mathrm{C}$ and ${ }^{1} \mathrm{H}$ gyromagnetic ratios. The proton spectrum of ethylene glycol has two significant peaks arising from its hydroxyl 
$(\mathrm{OH})$ and methylene $\left(\mathrm{CH}_{2}\right)$ groups. This poses a challenge for the phase-based dual-echo method. The echo time was therefore chosen to match the chemical shift difference of the two peaks ( $\sim 212 \mathrm{~Hz}$ at room temperature), to enable calculation of the accumulated phase based on two in-phase measurements.

Center frequency calibration on ${ }^{13} \mathrm{C}$ was performed manually by non-selective excitation. However, with the non-uniform receive profile of the ${ }^{13} \mathrm{C}$ array, this resulted in a center frequency that did not match the ${ }^{1} \mathrm{H}$ center frequency based on non-selective excitation with the body coil. The $\mathrm{B}_{0}$-field mapped at the proton frequency was therefore shifted to compensate. RF transmit power was calibrated with an automated Bloch-Siegert phase shift method (27).

\section{IN VIVO EXPERIMENTS}

The different sensitivity calibration schemes were tested in vivo in a hyperpolarized [1${ }^{13} \mathrm{C}$ ]pyruvate kidney study of a healthy female Danish domestic pig weighing $40 \mathrm{~kg}$. The pig received three hyperpolarized injections to acquire data with the fully sampled 3D stack-ofspirals and the two undersampled versions, respectively.

The procedure was similar to a previously reported $(28,29)$. In short, the pig was anesthetized with an initial dose of IV propofol $12 \mathrm{mg}$, intubated, and mechanically ventilated using a $60 \%$ $\mathrm{O}_{2}$-air mix using a commercially available Avance respirator system with a built- in $\mathrm{CO}_{2}$ and $\mathrm{O}_{2}$ gas monitoring unit (GE Healthcare, Brøndby, Denmark). Anesthesia was maintained with IV propofol $0.4 \mathrm{mg} / \mathrm{kg} / \mathrm{h}$ and fentanyl $8 \mu \mathrm{g} / \mathrm{kg} / \mathrm{h}$. By ultrasound guidance, sheaths were placed in femoral arteries and veins on the left and right side for blood sampling, monitoring of arterial blood pressure, and administration of hyperpolarized $\left[1-{ }^{13} \mathrm{C}\right]$ pyruvate. The animal experiment was done in accord with relevant laws and ethics under permission from The Animal Experiments Inspectorate in Denmark.

$641 \mathrm{mg}\left[1{ }^{13} \mathrm{C}\right]$ pyruvate with $15 \mathrm{mM}$ of $\mathrm{AH} 111501$ was polarized for $2.5 \mathrm{~h}$ in a SPINlab polarizer (GE Healthcare, Brøndby, Denmark). Dissolution was performed with $29 \mathrm{~mL}$ of de-ionized water into a receiver syringe, containing $13 \mathrm{~mL}$ of $360 \mathrm{mM}$ of sodium hydroxide and $181 \mathrm{mM}$ of tris(hydroxymethyl)aminomethane for neutralization of the pyruvic acid. Approximately $25 \mathrm{~mL}$ of $250 \mathrm{mM}$ pyruvate $(0.63 \mathrm{~mL} / \mathrm{kg})$ was injected IV, followed by a $20-\mathrm{mL}$ saline flush. Total injection took approximately $10 \mathrm{~s}$. Acquisition was started at the beginning of saline injection.

${ }^{13} \mathrm{C}$ MR acquisition was done with a TR of $90 \mathrm{~ms}$ per excitation, and two averages per excitation, amounting to an effective TR per metabolite volume of $5.4 \mathrm{~s}$ for the fully sampled 
acquisition and half that time, $2.7 \mathrm{~s}$, for the undersampled acquisitions. Signals from pyruvate and lactate were acquired in an interleaved fashion. The effective temporal resolutions for both pyruvate and lactate were hereby $10.8 \mathrm{~s}$ for the fully sampled acquisition, and $5.4 \mathrm{~s}$ for the undersampled acquisitions. The same constant flip angle of $5^{\circ}$ was used for both pyruvate and lactate excitations. This amounts to an effective flip angle of $26.9^{\circ}$ per volume for the fully sampled acquisition and $19.2^{\circ}$ for the undersampled acquisitions. ${ }^{13} \mathrm{C}$ MRI acquisition was done with free breathing.

The $\mathrm{G}_{\mathrm{z}}$-gradient of the SPSP pulse was scaled to limit excitation outside the encoded FOV, at a cost of non-uniform excitation efficiency (see Figure 1b). This trade-off was made, as wraparound effects in the full FOV for parallel imaging represent a significant source of artifacts.

A gadolinium-doped ${ }^{13} \mathrm{C}$-urea phantom was used for both center frequency referencing and transmit power calibration prior to injection. Each ${ }^{13} \mathrm{C}$ MRI stack-of-spirals acquisition was followed by acquisition of $\mathrm{a}^{13} \mathrm{C}$ spectrum to evaluate the center frequency estimate and adjust it if needed before the next acquisition.

Main field inhomogeneity was estimated at the ${ }^{1} \mathrm{H}$ frequency using the body coil and the scanner software IDEAL-IQ (GE Healthcare); a 3D imaging sequence used to estimate parameter maps from a single breath-hold acquisition. Similar to the phantom experiments, the $\mathrm{B}_{0}$-field was shifted to account for a mismatch of ${ }^{1} \mathrm{H}$ and ${ }^{13} \mathrm{C}$ center frequencies.

To provide anatomic references for the ${ }^{13} \mathrm{C}$ images, additional proton acquisitions were made with the body coil during single breath-hold. These included two multi-slice GRE acquisitions in the axial and coronal scan planes, respectively $\left(\mathrm{TR} / \mathrm{TE}=300 / 4.5 \mathrm{~ms}\right.$, flip angle $=30^{\circ}, \mathrm{FOV}=$ $40 \times 40 \mathrm{~cm}^{2}$, slice thickness $=1 \mathrm{~cm}$, matrix size $\left.128 \times 128\right)$.

Finally, to estimate renal perfusion, a DCE-MRI acquisition was performed after administration of gadolinium at a dose of $0.4 \mathrm{~mL} / \mathrm{kg}\left(\right.$ Clariscan $\left.^{\mathrm{TM}}\right)$. Acquisition was done using the scanner software DISCO; a dual-echo 3D spoiled gradient-echo sequence. One acquisition was done before contrast injection for baseline estimation. After contrast injection, a dynamic series of 49 images was acquired $\left(\mathrm{TR} / \mathrm{TE}=3.8 / 1.7 \mathrm{~ms}\right.$, flip angle $=12^{\circ}, \mathrm{FOV}=34 \times 34 \times 5.4 \mathrm{~cm}^{3}$, matrix size $=$ $160 \times 128 \times 12)$.

RECONSTRUCTION

All reconstructions were performed in MATLAB. 
For phantom and in vivo reconstructions based on phantom pre-calibration, sensitivity profiles were extracted from the calibration data by separate linear fitting of the magnitude and phase images. Magnitude data were fitted by third order polynomial fitting (including interactions) of their natural logarithm transform. Phase data were first calculated relative to one coil element, then unwrapped and finally fitted by a first order polynomial (including interactions) with a weight function, $w=1 /(1+\mid$ residual $\mid)$, to make the fitting more robust. The fitted functions were evaluated at the needed positions, which entailed both inter- and extrapolation. This ensured that the phantom pre-calibrated sensitivities could be used for reconstructions with higher resolution and for reconstructions, where the object size exceeded that of the phantom.

For the simulated data, all phantom pre-calibrated reconstructions were based on sensitivity profiles extracted from the highest simulated SNR level. With no inter- or extrapolation needed in this case, sensitivity profiles were extracted by performing edge-preserving smoothing using Perona-Malik Anisotropic Diffusion filtering (30). The degree of smoothing and anisotropy of the filter is controlled by two separate parameters set relative to the maximum signal and SNR level; i.e. the higher the maximum signal, the greater the anisotropy of the filter, and the higher the SNR, the less smoothing.

For reconstructions of auto-calibrated data, sensitivity profiles were also extracted by means of edge-preserving smoothing, but based on the reconstructed images of the low resolution fully sampled k-space center. The sensitivity profiles were subsequently resized to match the final reconstructed resolution using bicubic interpolation. For the in vivo auto-calibration reconstruction, sensitivity extraction was based on the time integral of the fully sampled, lowresolution pyruvate images.

Theoretical sensitivity maps were used directly in the simulated reconstructions, as these also represented the actual simulated profiles. For the phantom and in vivo reconstructions, however, the phase maps of the simulated profiles needed to be shifted to represent the actual coil phases affected by hardware-induced phase delays. The needed phase shift for each coil element was found by least-squares minimization of the phase difference to the phantom precalibration maps. The scaling of each coil element, needed to account for differences in receiver gains, was also found by least-squares fitting.

Off-resonance correction due to main field inhomogeneity was performed for both simulated, phantom and in vivo data. As the imaging sequence acquires data in 3D k-space, the implementation of inhomogeneity correction required full 3D gridding. Routines based on the 
NUFFT (non-uniform fast Fourier transform) were given by "gpuNUFFT - Open-Source GPU Library for 3D Gridding" on GitHub (31), which provides a 3D interface to Jeffrey Fessler's MIRT Toolbox (32), and enables NUFFTs to be performed on the GPU. GPU computing was not exploited in the current implementation, but should be easy to include to reduce reconstruction time. Static field off-resonance correction was done as described in Sutton et al. (33), by applying its $2 \mathrm{D}$ implementation from the MIRT Toolbox to the 3D reconstruction framework provided by gpuNUFFT. The inhomogeneity correction method performs fast, iterative, fieldcorrected reconstruction by utilizing a time-segmented approximation to the MR signal equation. The number of time-segments needed for accurate correction is automatically estimated based on readout duration and a histogram analysis of the provided $\mathrm{B}_{0}$-field map. After the final iteration in the 3D gridding routine, the gridded k-space data were masked with a region of support for the stack-of-spirals trajectory. The mask was filtered with a 3D Gaussian smoothing kernel to reduce ringing artifacts in the final image.

For the undersampled 3D reconstructions, SENSE was implemented as an iterative reconstruction (34) by means of the conjugate gradient (CG) algorithm with code from the above-mentioned GitHub toolboxes. To reduce ill-conditioning, all sensitivities were masked before they were given as input to the CG-SENSE algorithm. This includes the prior knowledge of the correct signal values outside the masks being zero (5).

To also explore optimal reconstruction of fully sampled data with respect to coil-combination, different combination methods were compared: sum-of-squares coil-combination, autocalibrated coil-combination, and coil-combination based on phantom and theoretical precalibrated maps.

\section{RESULTS}

\section{SIMULATIONS}

Figure $3 a$ summarizes the effects of noise on the different simulation reconstructions. SNR values represent maximum SNR for the uncombined coil images. Structural similarity to the true sample is highest for the reconstructions using the theoretical pre-calibrated sensitivities, which was expected as these also represent the true sensitivities used in the simulation. The fact that the structural similarity of the reconstructed undersampled data is similar to that of the fully sampled data, indicates minimal g-factor penalty. 
At high SNR levels $(>20)$ the similarity to the true sample is stable for all reconstructions. As SNR decreases to less than 10, SSIM values fall drastically for all reconstructions. At SNR $<10$ central parts of the object far from the coil elements were at the noise-level.

The SSIM values for the fully sampled sum-of-squares reconstructions stand out in the graph. One reason is that this reconstruction does not include intensity correction. Intensity modulation decreases structural similarity to the true object, although it does not decrease SNR.

Figure $3 b$ shows the central sagittal slice of the reconstructed volume at three different SNRlevels to aid the interpretation of the SSIM metric in context of the graph in Figure 3a. From these images, it is seen that the lower SSIMs of the phantom pre-calibrated reconstructions, compared to those of the theoretical pre-calibrated reconstructions, might be partially explained by over-smoothing at the edges, despite the efforts to avoid this.

\section{PHANTOM EXPERIMENTS}

Supporting Information Figure S2 shows the fully sampled phantom data reconstructed using four different coil-combination methods. The sum-of-squares combined images show a lower contrast between background noise and signal, which is explained by SNR-dependent bias introduced by performing magnitude-based coil-combination (35). The three reconstructions using sensitivity maps are similar, which indicate successful registration of the pre-calibrated maps.

Figure 4 shows a representative sagittal slice from the reconstructed volume for different sampling patterns and reconstructions. The last column shows images reconstructed using the theoretical pre-calibration maps, and although the two bottom figures were reconstructed from undersampled data, all three figures are close to identical. Reconstructions with phantom precalibrated and auto-calibrated maps (center column) were all successful in resolving aliasing artifacts.

Figure 5 shows representative slices for the uniformly undersampled trajectory reconstructed with the theoretical sensitivities. The full volume reconstruction can be found in Supporting Information Figure S3. Figure 5 shows the sum-of-squares reconstruction, and the reconstruction with and without intensity correction. The figure also shows off-resonance artifacts in context of the measured $\mathrm{B}_{0}$-field, as well as the correction of these. The edges at the signal voids appear sharp, which indicates both a satisfactory $\mathrm{B}_{0}$-field estimation and registration, as well as a well-functioning correction algorithm. 
The intensity corrected reconstruction in Figure $5 d$ represents the best result of the phantom experiment among the reconstructions of undersampled data. Compare these images to the digital phantom in Supporting Information Figure S1.

\section{IN VIVO EXPERIMENTS}

The physiological state of the pig during the scan session is summarized in Supporting Information Table S1.

Figure 6 shows temporal averages for two coronal slices of the 3D volume covering the kidneys, illustrating different reconstruction methods. The three different acquisition schemes applied after the three individual hyperpolarized injections are indicated by braces to the left. For the uniform undersampling scheme, the sum-of-squares reconstruction reveals a convenient aliasing pattern for the kidneys, since these are close to free from aliasing artifacts. The majority of aliasing artifacts occurring elsewhere were resolved by the CG-SENSE reconstructions. However, some aliasing artifacts were left unresolved (indicated by the white arrows). For the auto-calibration acquisition scheme, the undersampling pattern resulted in more blurry aliasing artifacts. Possibly due to unresolved aliasing, renal structures are slightly less clear in the autocalibrated reconstructions, compared to the pre-calibrated reconstructions.

The lactate images in Figure 6 demonstrate the benefit of performing sensitivity-based reconstructions for multi-channel datasets with low SNR. This is illustrated by the increased contrast between signal and background noise compared to the sum-of-squares reconstructions.

Figure 7 shows the metabolite dynamics for a single slice of the kidneys for phantom and theoretical pre-calibrated reconstructions of both uniformly undersampled and fully sampled datasets. Unlike the images shown in Figure 6, these images were corrected for coil-profile intensity variations to give uniform images. Structural information is provided by both undersampled and fully sampled acquisitions through a higher signal of pyruvate and lactate in the renal cortices than in the medullas. Application of intensity correction results in similar signal intensities for the left and right kidney, as expected for a healthy pig. This was supported by the DCE-MRI measurement, which showed equal and normal perfusion levels.

Figure 8 shows the metabolite dynamics averaged across the renal cortices and demonstrate better capture of the steep upslope of the pyruvate curve for the higher temporal resolution of the undersampled acquisitions. 
Supporting Information Videos S1-3 show a maximum intensity projection of the pyruvate time integrated signal from different view angles, the pyruvate dynamics in axial and coronal view, respectively. All for the theoretical pre-calibrated reconstruction.

\section{DISCUSSION}

The main finding of this study is that theoretically pre-calibrated sensitivities provides the highest image quality for parallel imaging acquisition in hyperpolarized ${ }^{13} \mathrm{C} \mathrm{MRI}$ using a fixed receive coil geometry. In this study, pre-calibrated sensitivities enabled reconstruction of accelerated 3D volumes of renal metabolism. This is important, since current non-accelerated methods typically do not allow for sufficient temporal and spatial resolution to map abdominal organ metabolic and functional heterogeneity - a key requirement for clinical translation of hyperpolarized MR $(36,37)$.

Results from the simulations verified the reconstruction pipeline: Simulated static field inhomogeneity could be corrected and aliasing artifacts from undersampling could be resolved. The simulations also showed higher image quality for uniformly undersampled data using precalibrated maps, compared to the auto-calibration reconstructions. This is not surprising considering the higher undersampling rate in the outer parts of k-space for the auto-calibration scheme.

The comparison of coil-combination methods for fully sampled data (Supporting Information Figure S2) demonstrated an image quality benefit from using coil sensitivities in agreement with literature $(38,39)$. In cases where sensitivities are not known, auto-calibrated coil-combination demonstrated image quality similar to pre-calibrated coil-combined images. This is useful for phantom studies and coil array testing, both due to the higher image quality compared to sumof-squares combination, and because sensitivity-based coil-combination results in minimal noise-bias, independent of the number of coil elements. For phantoms with signal nonuniformity and for in vivo imaging, auto-calibrated coil sensitivities need to be based on the sumof-squares normalized image to divide out object contrast. This will still result in increased image quality, however, without intensity correction $(39,40)$.

The phantom results share a trend with the simulations, in the sense that theoretical precalibrated reconstructions resulted in the highest image quality, and auto-calibrated reconstructions resulted in the lowest image quality. The success of the theoretical precalibrated sensitivities relies on fixed coil geometry and the sensitivity profiles being minimally affected by load at the relatively low carbon frequency at $3 \mathrm{~T}$. The slightly lower image quality of 
the auto-calibrated reconstruction is consistent with the simulation. For an in vitro, stationary experimental setup pre-calibration methods are expected to outperform auto-calibration methods. The strength of auto-calibration methods is usually demonstrated in the presence of motion, where a closer registration of the reference data to the target data may yield better results (5).

For the in vivo study, where motion was present, the auto-calibrated reconstruction did, however, not prove advantageous compared to the pre-calibrated reconstructions. But as the auto-calibrated reconstruction was based on sensitivity map extraction from the pyruvate data summed through time, motion represents as big an issue as for the pre-calibrated reconstructions. It cannot be ruled out that a GRAPPA-like reconstruction could have provided a more robust reconstruction. However, GRAPPA kernel estimation would similarly require timeaveraged data or data from a maximum SNR time point, and would hereby likely be equally motion-sensitive.

Parallel imaging reconstruction was not able to resolve all artifacts for any of the undersampled reconstructions of the in vivo data. For the reconstruction based on phantom pre-calibration, this could be related to imperfect extrapolation of the phantom maps: As the pig covered a larger part of the FOV compared to the phantom, parts of the sensitivity map were estimated based on extrapolated values, making them less reliable. Nevertheless, the theoretical pre-calibrated reconstruction also had residual aliasing artifacts, mainly seen as fold-in of the blood vessels. With the strong signal intensity of the blood vessels combined with their central location within the coil (where profiles are least unique), these represented a difficult parallel imaging reconstruction task. Fortunately, though, for this experiment the artifacts did not interfere with the kidneys, being the target organ of the study.

Intensity corrected images, as those shown in Figure 7, produce more readily interpretable metabolite images. This together with the higher image quality of sensitivity-based coilcombination, represent a strong argument for using sensitivity profiles as a prior in reconstruction of multi-channel hyperpolarized data, when reliable sensitivity profiles are available.

The main strengths of using pre-calibrated maps for parallel imaging acquisition and reconstruction for hyperpolarized MRI have here been demonstrated through volumetric acquisition with increased temporal resolution. Whether this leads to better accuracy in kinetic modeling or other characteristics of the metabolite dynamics depends on the specific situation. 
Weaknesses of the experimental setup in this study, include the fact that the coil setup was not designed to be easily combined with a proton receive array, resulting in low quality proton scans. Another weakness results from the use of SPSP excitation: When acquiring data over large volumes, shimming becomes more challenging, and so does off-resonance excitation artifacts. In a recent study by Lau et al. (41) such artifacts were mitigated by increasing the spectral passband of the SPSP pulse and acquiring multiple echoes to resolve signal contamination in the reconstruction, similar to IDEAL (42). Besides being sensitive to offresonance, the used SPSP pulse was also rather long. A long echo time leads to loss of SNR, but a recent study by Chen et al. (43) also found that a long echo time for hyperpolarized experiments can cause errors in the estimated kinetics due to the shorter T2* of lactate compared to pyruvate. Therefore, future improvements should include a better SPSP design and/or IDEAL reconstruction.

Other potential improvements include the acceleration scheme, which for this study did not make optimal use of the geometry of the receive array. A future scheme should also be accelerated in the axial plane, while reducing through-plane acceleration, for decreased residual aliasing, increased temporal resolution, and more measured metabolites. The approach could also be tested with a different receive array with fixed geometry. While the coil geometry is expected to greatly influence the success of the parallel imaging experiment, a different coil geometry is not expected to change the conclusions with respect to sensitivity calibration.

The focus of this study is on hyperpolarized ${ }^{13} \mathrm{C}$ imaging on $3 \mathrm{~T}$, the dominant field strength for large animal and human hyperpolarized applications. We expect an upper limit for the field strength, at which sample loading becomes dominant, where the proposed method will no longer be applicable.

The in vivo experiments in this study were performed under circumstances similar to human hyperpolarized imaging. With a fixed geometry, multi-channel ${ }^{13} \mathrm{C}$ coil and a stationary target organ, such as the kidneys or the brain, it is therefore expected that parallel imaging using theoretical pre-calibration would also provide the best results for human imaging with freebreathing.

\section{CONCLUSIONS}

This study tested different approaches to sensitivity calibration for parallel imaging reconstruction of hyperpolarized ${ }^{13} \mathrm{C}$ MRI using SENSE. The auto-calibration approach did not prove advantageous in simulations, phantom experiments, or in vivo. Pre-calibrated parallel 
imaging reconstructions, on the other hand, resulted in high quality images both for phantom and in vivo experiments, though the reconstruction was more challenged and less successful in the in vivo case. From a practical point of view, pre-calibrated parallel imaging is also preferred, since it enables the use of standard sequences with reduced FOV for image acceleration. Precalibration using theoretically inferred maps gave better results for both the phantom and the in vivo study and does not require a long phantom scan nor extrapolation, only spatial registration.

Overall this study demonstrated improved image quality for volumetric hyperpolarized ${ }^{13} \mathrm{C} M R \mathrm{I}$ acquisition by means of parallel imaging using SENSE and theoretical pre-calibrated sensitivity maps.

\section{ACKNOWLEDGEMENTS}

This work has been partly funded by the Independent Research Fund Denmark (DFF - 400500531) and the Danish National Research Foundation (DNRF124).

\section{REFERENCES}

1. Ardenkjær-Larsen JH, Fridlund B, Gram A, et al. Increase in signal-to-noise ratio of $>10,000$ times in liquid-state NMR. Proc. Natl. Acad. Sci. U. S. A. 2003;100:10158-10163 doi: 10.1073/pnas.1733835100.

2. Golman K, Zandt RI, Lerche MH, Pehrson R, Ardenkjær-Larsen JH. Metabolic imaging by hyperpolarized $13 \mathrm{C}$ magnetic resonance imaging for in vivo tumor diagnosis. Cancer Res. 2006;66:10855-10860 doi: 10.1158/0008-5472.CAN-06-2564.

3. Schroeder MA, Clarke K, Neubauer S, Tyler DJ. Hyperpolarized magnetic resonance: A novel technique for the in vivo assessment of cardiovascular disease. Circulation 2011;124:15801594 doi: 10.1161/CIRCULATIONAHA.111.024919.

4. Cunningham CH, Lau JY, Chen AP, et al. Hyperpolarized 13C Metabolic MRI of the Human Heart: Initial Experience. Circ. Res. 2016;119:1177-1182 doi:

10.1161/CIRCRESAHA.116.309769.

5. Larkman DJ, Nunes RG. Parallel magnetic resonance imaging. Phys. Med. Biol. 2007;52:R15-55 doi: 10.1088/0031-9155/52/7/R01.

6. Arunachalam A, Whitt D, Fish K, et al. Accelerated spectroscopic imaging of hyperpolarized C-13 pyruvate using SENSE parallel imaging. NMR Biomed. 2009;22:867-873 doi: 
10.1002/nbm.1401.

7. Pruessmann KP, Weiger M, Scheidegger MB, Boesiger P. SENSE: Sensitivity encoding for fast MRI. Magn. Reson. Med. 1999;42:952-962 doi: 10.1002/(SICI)15222594(199911)42:5<952::AID-MRM16>3.0.CO;2-S.

8. Griswold MA, Jakob PM, Heidemann RM, et al. Generalized Autocalibrating Partially Parallel Acquisitions (GRAPPA). Magn. Reson. Med. 2002;47:1202-1210 doi: 10.1002/mrm.10171.

9. Feng Y, Gordon JW, Shin PJ, et al. Development and testing of hyperpolarized 13C MR calibrationless parallel imaging. J. Magn. Reson. 2016;262:1-7 doi: 10.1016/j.jmr.2015.10.018.

10. Gordon JW, Hansen RB, Shin PJ, Feng Y, Vigneron DB, Larson PE. 3D Hyperpolarized C13 EPI with Calibrationless Parallel Imaging. J. Magn. Reson. 2018;289:92-99 doi: 10.1016/j.jmr.2018.02.011.

11. Shin PJ, Larson PE, Ohliger MA, et al. Calibrationless parallel imaging reconstruction based on structured low-rank matrix completion. Magn. Reson. Med. 2014;72:959-70 doi: 10.1002/mrm.24997.

12. Ohliger MA, Larson PE, Bok RA, et al. Combined parallel and partial Fourier MR reconstruction for accelerated 8-channel hyperpolarized carbon-13 in vivo magnetic resonance Spectroscopic imaging (MRSI). J. Magn. Reson. Imaging 2013;38:701-713 doi: 10.1002/jmri.23989.

13. Jiang W, Lustig M, Larson PE. Concentric rings k-space trajectory for hyperpolarized $13 \mathrm{C}$ MR spectroscopic imaging. Magn. Reson. Med. 2016;75:19-31 doi: 10.1002/mrm.25577.

14. Tropp J, Lupo JM, Chen AP, et al. Multi-channel metabolic imaging, with SENSE reconstruction, of hyperpolarized [1-13C] pyruvate in a live rat at 3.0 tesla on a clinical MR scanner. J. Magn. Reson. 2011;208:171-177 doi: 10.1016/j.jmr.2010.10.007.

15. Dominguez-Viqueira W, Geraghty BJ, Lau JY, Robb F, Chen AP, Cunningham CH. Intensity correction for multichannel hyperpolarized $13 \mathrm{C}$ imaging of the heart. Magn. Reson. Med. 2016;75:859-865 doi: 10.1002/mrm.26042.

16. Wiesinger F, Van de Moortele PF, Adriany G, De Zanche N, Ugurbil K, Pruessmann KP. Potential and feasibility of parallel MRI at high field. NMR Biomed. 2006;19:368-378 doi: 10.1002/nbm.1050. 
17. Kohler SJ, Yen Y-F, Wolber J, et al. In Vivo 13 Carbon Metabolic Imaging at 3T With Hyperpolarized 13 C-1-Pyruvate. Magn. Reson. Med. 2007;58:65-69 doi: 10.1002/mrm.21253.

18. Köllisch U. Advanced Cardiac Magnetic Resonance Imaging with Hyperpolarized 13C Acetate und Pyruvate [dissertation]. Munich, Germany: Technischen Universität München; 2015.

19. Larson PE, Kerr AB, Chen AP, et al. Multiband excitation pulses for hyperpolarized 13C dynamic chemical-shift imaging. J. Magn. Reson. 2008;194:121-127 doi:

10.1016/j.jmr.2008.06.010.

20. Nelson SJ, Kurhanewicz J, Vigneron DB, et al. Metabolic Imaging of Patients with Prostate Cancer Using Hyperpolarized [1-13C] Pyruvate. Sci. Transl. Med. 2013;5:198ra108 doi: 10.1126/scitransImed.3006070.

21. Sánchez-Heredia JD, Hansen RB, Baron R, et al. Calibrated Coil Combination for FixedGeometry, Low-Frequency Coils with Application to Hyperpolarized 13C Measurements. In: Proc. Intl. Soc. Mag. Reson. Med. ; 2019. p. 1450.

22. Sánchez-Heredia JD, Johansen DH, Hansen RB, et al. Improved Decoupling for Low Frequency MRI Arrays using Non-conventional Preamplifier Impedance. IEEE Trans. Biomed. Eng. 2018 doi: 10.1109/TBME.2018.2881203.

23. Stöcker T, Vahedipour K, Pflugfelder D, Shah NJ. High-performance computing MRI simulations. Magn. Reson. Med. 2010;64:186-193 doi: 10.1002/mrm.22406.

24. Joe $\mathrm{E}$, Lee $\mathrm{H}$, Lee $\mathrm{J}$, et al. An indirect method for in vivo T2 mapping of [1-13C] pyruvate using hyperpolarized 13C CSI. NMR Biomed. 2017;30:1-8 doi: 10.1002/nbm.3690.

25. Wang Z, Bovik AC, Sheikh HR, Simoncelli EP. Image quality assessment: From error visibility to structural similarity. IEEE Trans. Image Process. 2004;13:600-612 doi: 10.1109/TIP.2003.819861.

26. Schneider E, Glover GH. Rapid in vivo proton shimming. Magn. Reson. Med. 1991;18:335347 doi: $10.1002 / \mathrm{mrm} .1910180208$.

27. Schulte RF, Sacolick LI, Deppe MH, et al. Transmit gain calibration for nonproton MR using the Bloch-Siegert shift. NMR Biomed. 2011;24:1068-1072 doi: 10.1002/nbm.1657. 
28. Laustsen C, Hansen ESS, Kjærgaard U, Bertelsen LB, Ringgaard S, Stødkilde-Jørgensen $\mathrm{H}$. Acute porcine renal metabolic effect of endogastric soft drink administration assessed with hyperpolarized [1-13c]pyruvate. Magn. Reson. Med. 2015;74:558-563 doi: 10.1002/mrm.25692.

29. Wigh Lipsø K, Hansen ESS, Tougaard RS, Laustsen C, Ardenkjær-Larsen JH. Renal MR angiography and perfusion in the pig using hyperpolarized water. Magn. Reson. Med. 2017;78:1131-1135 doi: 10.1002/mrm.26478.

30. Perona P, Malik J. Scale-Space and Edge detection using Anisotropic Diffusion. IEEE Trans. Pattern Anal. Mach. Intell. 1990;12:629-639 doi: 10.1109/34.56205.

31. Schwarzl A, Knoll F. gpuNUFFT - An Open-Source GPU Library for 3D Gridding with Direct Matlab Interface. GitHub. https://github.com/andyschwarzl/gpuNUFFT. Published 2014.

Accessed September 5, 2018.

32. Fessler JA. The Michigan Image Reconstruction Toolbox (MIRT).

http://web.eecs.umich.edu/ fessler/code/index.html. Published 2004. Accessed February 17, 2017.

33. Sutton BP, Noll DC, Fessler JA. Fast, iterative image reconstruction for MRI in the presence of field inhomogeneities. IEEE Trans. Med. Imaging 2003;22:178-188 doi: 10.1109/TMI.2002.808360.

34. Pruessmann KP, Weiger M, Bornert $P$, Boesiger P. Advances in Sensitivity Encoding With Arbitrary k-Space Trajectories. Magn. Reson. Imaging 2001;46:638-651 doi: 10.1002/mrm.1241.

35. Constantinides CD, Atalar E, Mcveigh ER. Signal-to-Noise Measurements in Magnitude Images from NMR Phased Arrays. Magn. Reson. Med. 1997;38:852-857 doi:

10.1002/mrm.1910380524.

36. Kurhanewicz J, Vigneron DB, Ardenkjær-Larsen JH, et al. Hyperpolarized 13C MRI: Path to Clinical Translation in Oncology. Neoplasia 2019;21:1-16 doi: 10.1016/j.neo.2018.09.006.

37. Laustsen C. Hyperpolarized renal magnetic resonance imaging: Potential and pitfalls. Front. Physiol. 2016;7:1-5 doi: 10.3389/fphys.2016.00072.

38. Roemer P, Edelstein W, Hayes CE. The NMR Phased Array. Magn. Reson. Med. 1990;16:192-225 doi: 10.1002/mrm.1910160203. 
39. Bydder M, Larkman DJ, Hajnal J V. Combination of signals from array coils using imagebased estimation of coil sensitivity profiles. Magn. Reson. Med. 2002;47:539-548 doi:

10.1002/mrm.10092.

40. Zhu Z, Zhu X, Ohliger MA, et al. Coil Combination Methods for Multi-Channel Hyperpolarized 13C Imaging Data from Human Studies. J. Magn. Reson. 2019 doi: 10.1016/j.jmr.2019.01.015.

41. Lau JY, Geraghty BJ, Chen AP, Cunningham CH. Improved tolerance to off-resonance in spectral-spatial EPI of hyperpolarized [1-13C]pyruvate and metabolites. Magn. Reson. Med. 2018;80:925-934 doi: 10.1002/mrm.27086.

42. Wiesinger F, Weidl E, Menzel MI, et al. IDEAL spiral CSI for dynamic metabolic MR imaging of hyperpolarized [1- 13C]pyruvate. Magn. Reson. Med. 2012;68:8-16 doi: 10.1002/mrm.23212.

43. Chen H-Y, Gordon JW, Bok RA, et al. Pulse sequence considerations for quantification of pyruvate-to-lactate conversion kPL in hyperpolarized 13C imaging. NMR Biomed. 2019 doi: 10.1002/nbm.4052. 
FIGURES
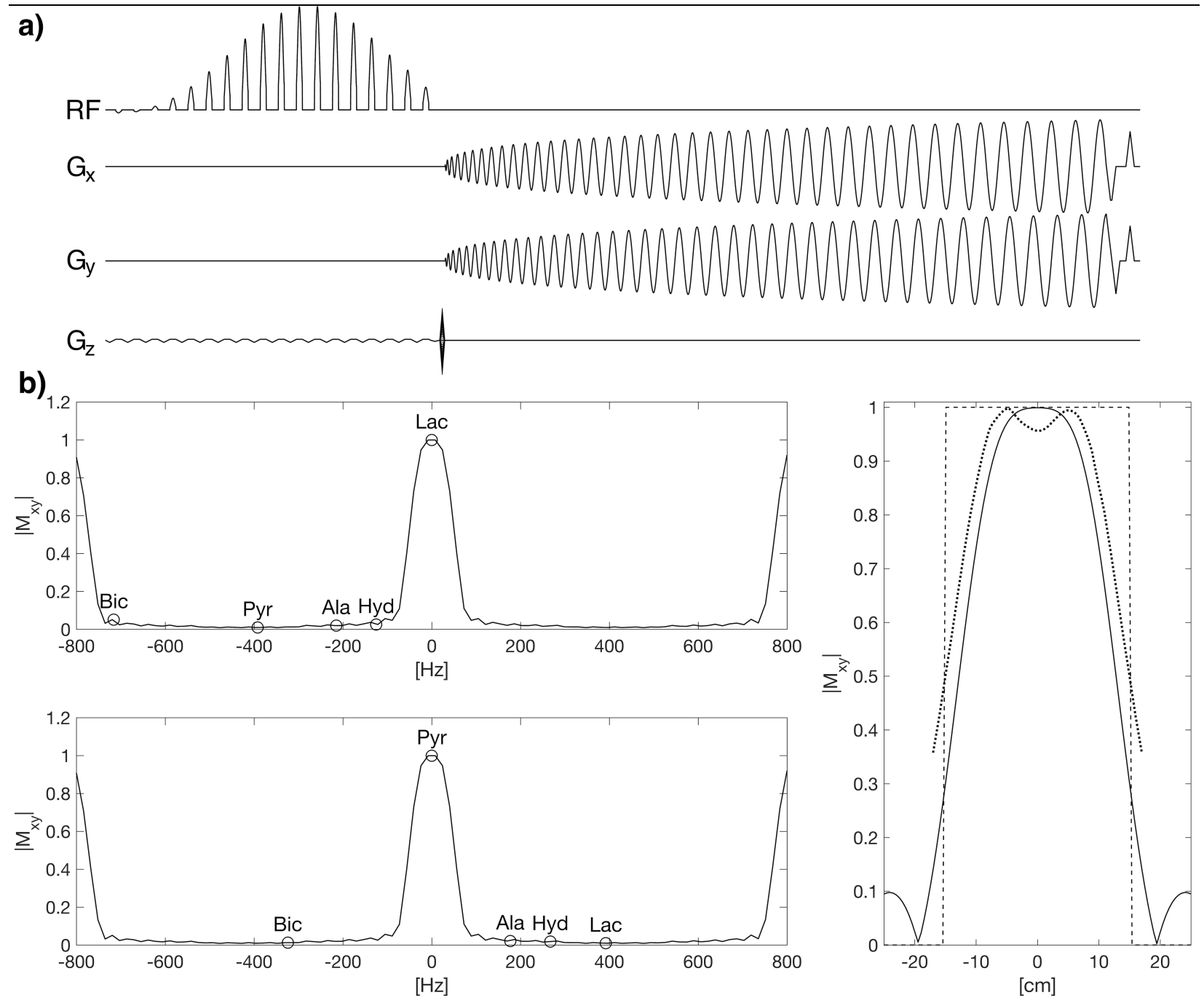

c)

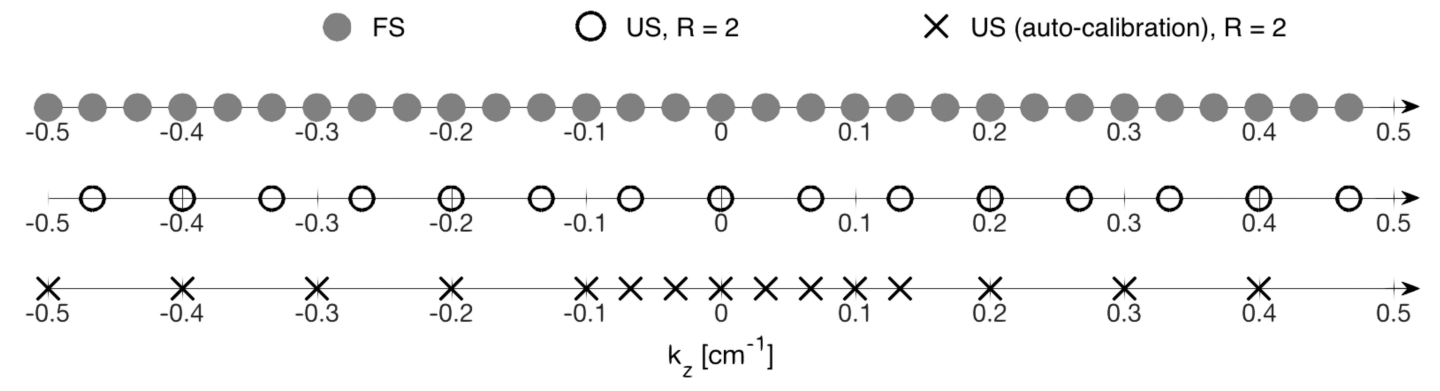

Figure 1: Pulse sequence and acquisition strategy. a) Pulse sequence diagram showing the spectral-spatial pulse with fly-back gradients (22.44 ms duration) followed by center-out ordered $z$ phase-encoding and $x y$ spiral readout. Total duration of the pulse sequence is $70 \mathrm{~ms}$. b) Spectral and spatial selectivity of the spectral-spatial pulse, shown with indications of the in vivo metabolite resonances and slab thickness (dashed bar) used for the in vivo experiments. The dotted curve in the spatial selectivity plot to the right, represents the relative sensitivity around the center of the used receive coil array (only simulated within a 34-cm region in $z$ ). c) Acquired samples along the $\mathrm{k}_{\mathrm{z}^{-}}$ direction for the trajectories illustrating the undersampling schemes. FS = fully sampled, US = undersampled. 


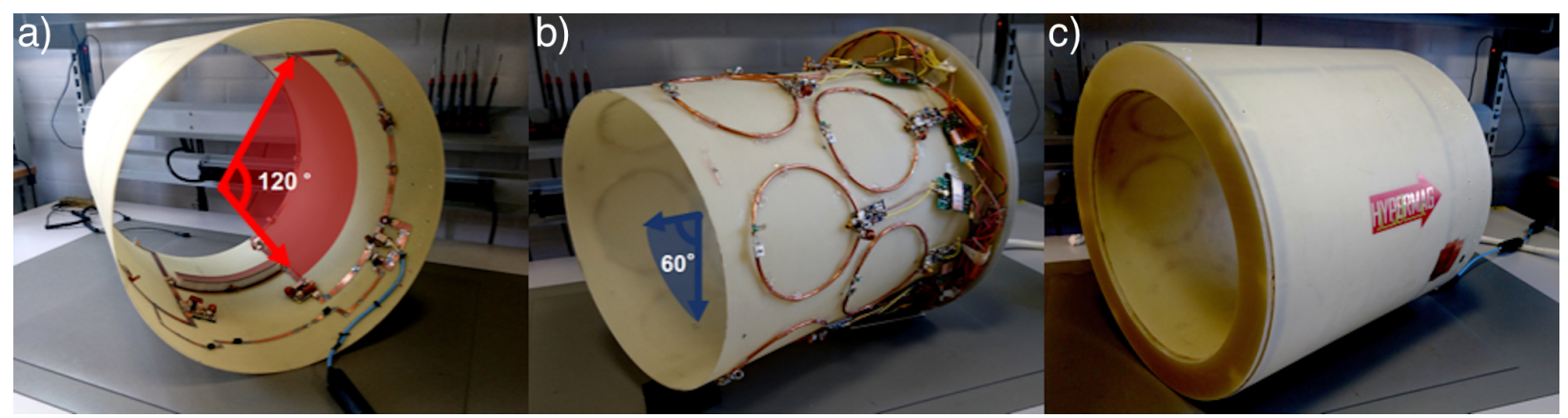

Figure 2: Custom-made coil geometry. a) Linear ${ }^{13} \mathrm{C}$ transmit coil. The coil is formed by two rectangular loops of $419 \times 300 \mathrm{~mm}^{2}$ (each covering $120^{\circ}$ of the coil perimeter). b) 12-element receive-only array, where each element has a diameter of $130 \mathrm{~mm}$. c) Integrated coil setup, where the two fiberglass cylinders are placed concentrically, such that their relative position is fixed.

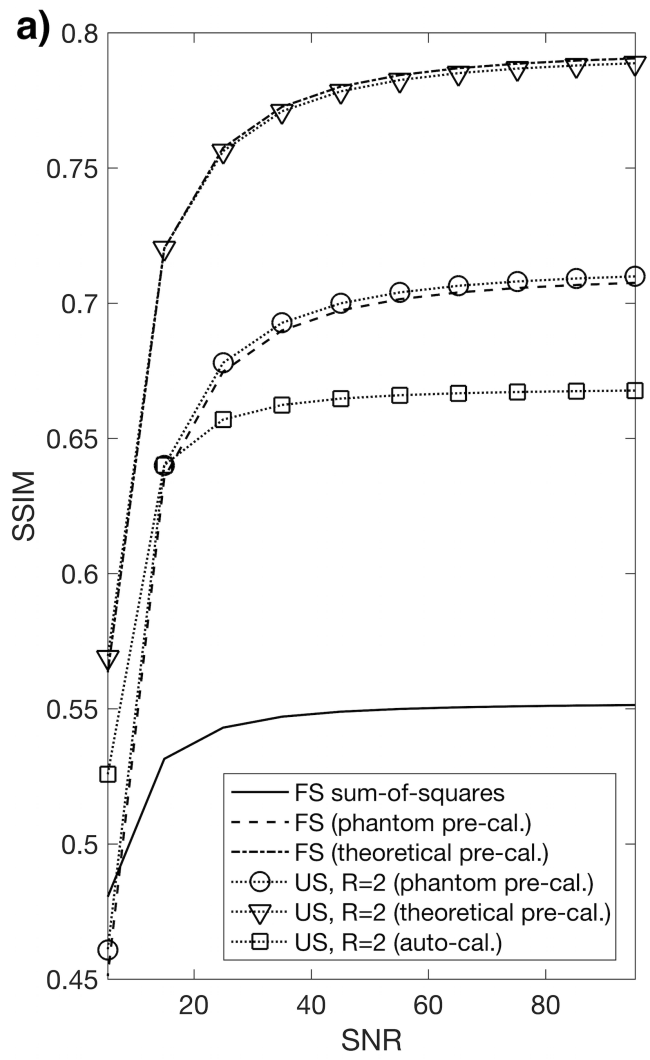

b)
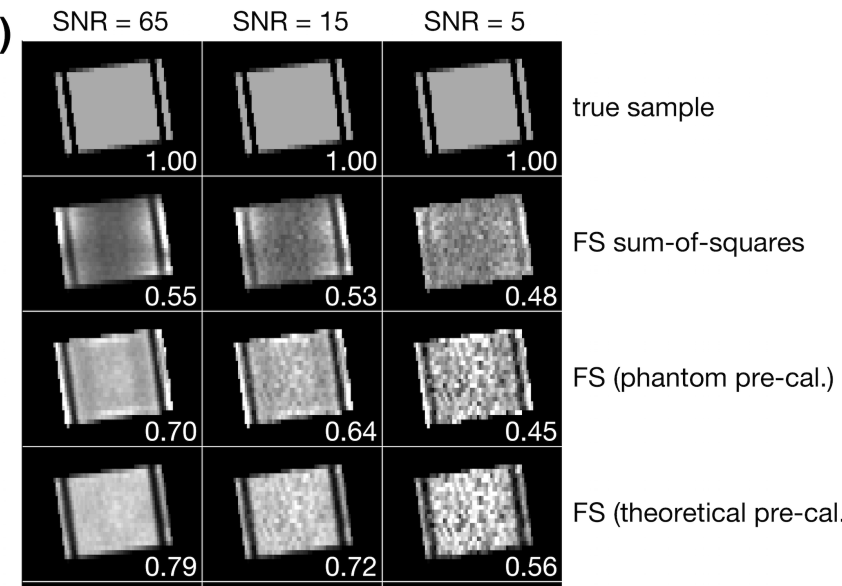

FS (theoretical pre-cal.)
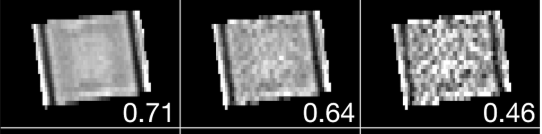

US, $\mathrm{R}=2$ (phantom pre-cal.)

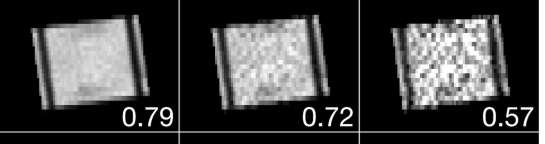

US, R=2 (theoretical pre-cal.)

Figure 3: Simulation results. a) Structural similarity, SSIM, as a function of maximum SNR of the uncombined image, for the different acquisition and reconstruction schemes. FS = fully sampled, US = undersampled. SSIM values represent the similarity of the intensity corrected images to the true sample. b) Example results for three different SNR levels represented by the central sagittal slice of the volume acquisition. Associated SSIM values are noted in the bottom right corner of each image. 


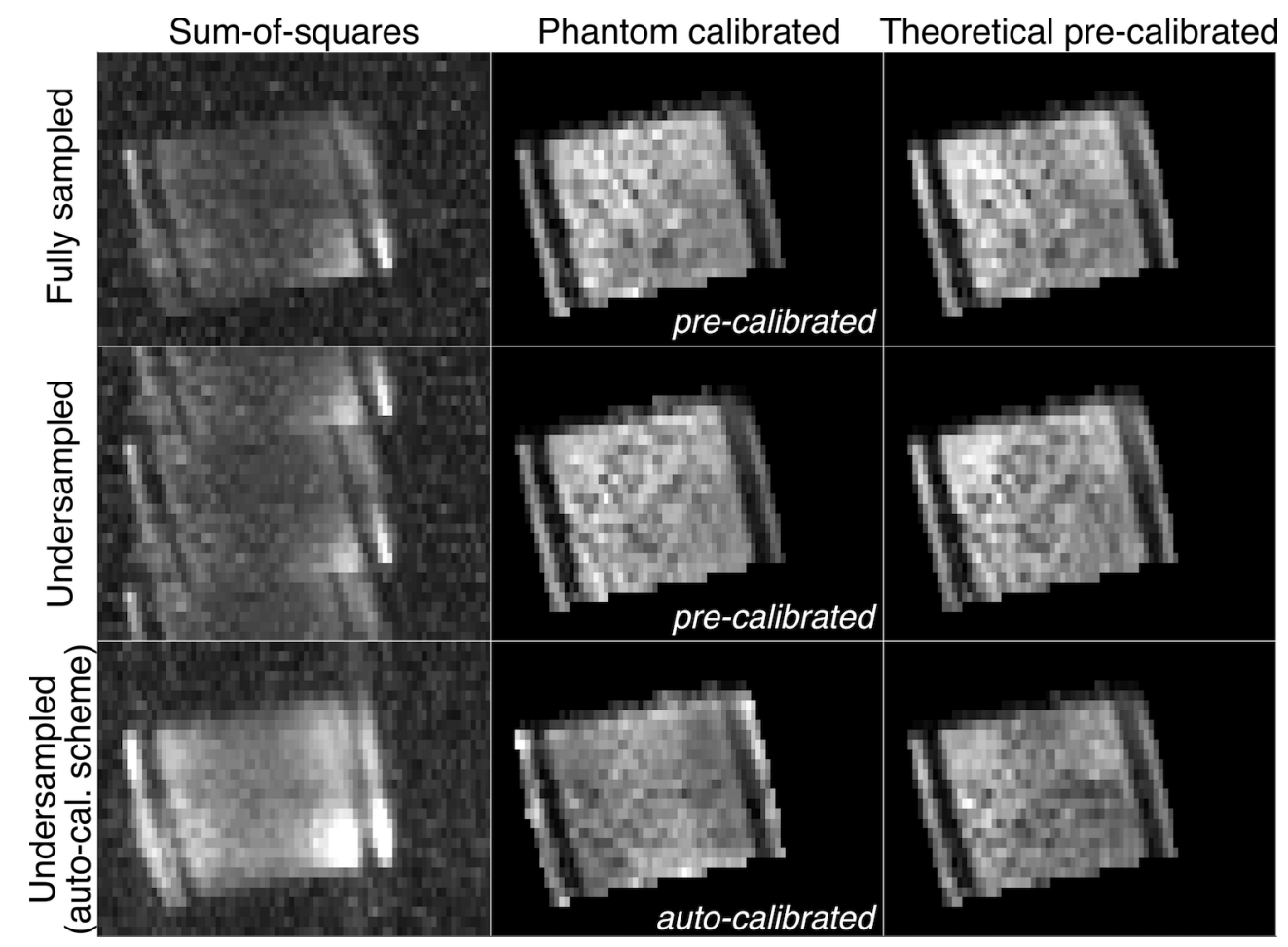

Figure 4: Central sagittal slice of the phantom data for different undersampling and reconstruction scenarios. The first column shows the sum-of-squares combined images. The second column shows reconstruction results using the pre-calibrated phantom sensitivities for the fully sampled and uniformly undersampled data, as well as reconstruction results for the auto-calibrated undersampling and reconstruction schemes. The last column shows the theoretical precalibrated reconstructions for all sampling schemes. 

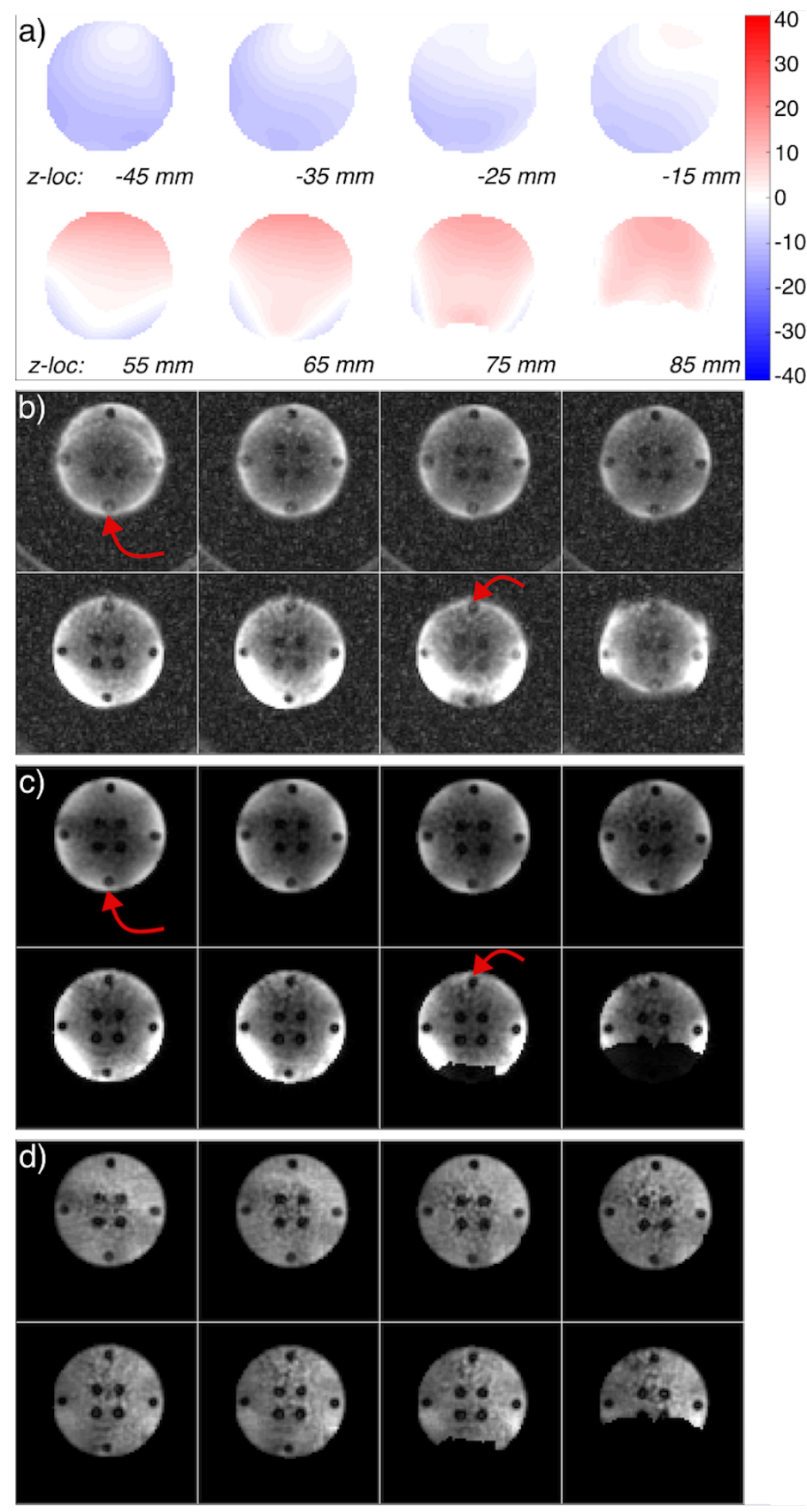

Figure 5: Axial slice representation of eight representative slices from the full acquisition volume for the uniformly undersampled data. Find all slices in Supporting Information Figure S3. a) Smoothed and masked $\mathrm{B}_{0}$-field map in units of $\mathrm{Hz}$, scaled and shifted to the ${ }^{13} \mathrm{C}$ frequency. b) Undersampled data after sum-of-squares combination. c) CGSENSE reconstruction using theoretical sensitivities and inhomogeneity correction. The red arrows point to two regions off-resonance (see a)), which led to distortions in b), but were successfully corrected for in c). d) The reconstruction in c) after intensity correction. 


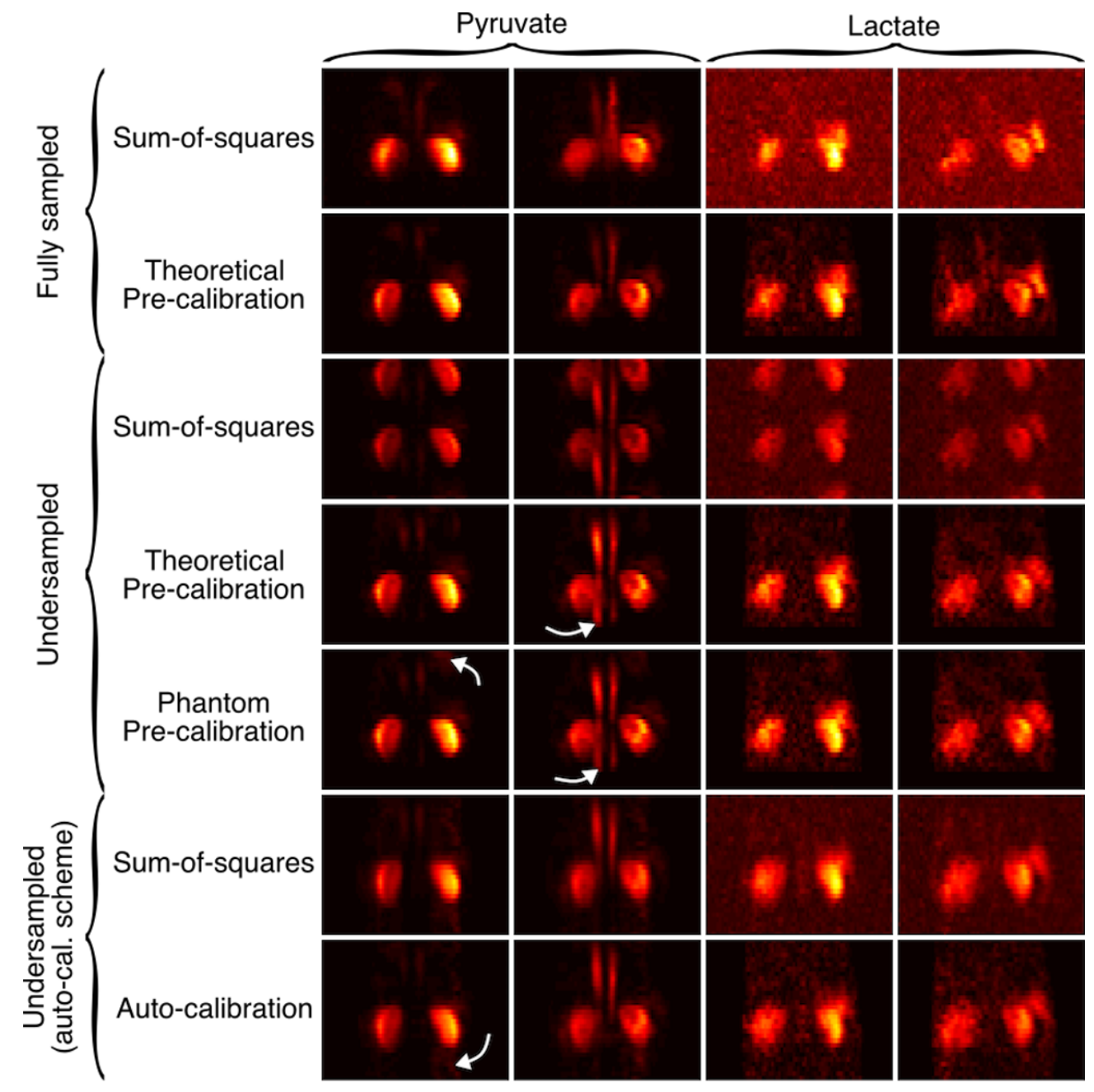

Figure 6: Two slices summed through time showing pyruvate uptake and lactate metabolism in the kidneys for different acquisition and reconstruction schemes. The images are from a slightly oblique scan plane (close to coronal) to best show both kidneys. All pyruvate and lactate images are, separately, shown on the same color scale after multiplying the fully sampled images by two, to account for half the number of acquired time points. Note that no signal mask was used for the sum-of-squares reconstructions, however the applied mask for the sensitivity-based reconstructions only suppresses noise in the left-right regions of the images. 


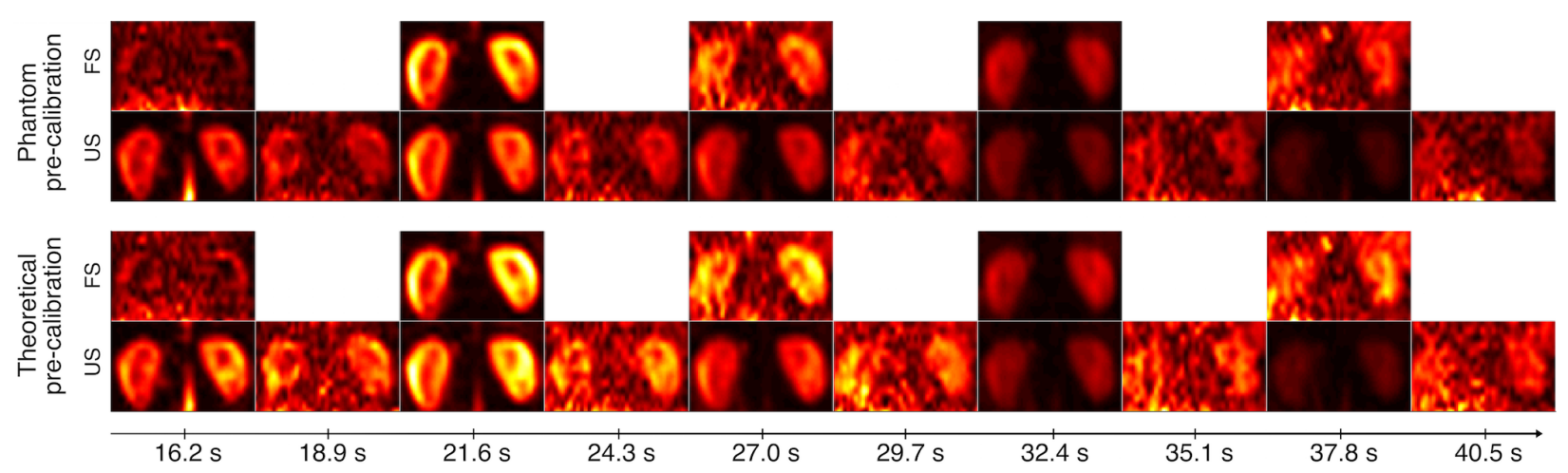

Figure 7: Pyruvate and lactate dynamics in the kidneys for pre-calibrated, intensity corrected reconstructions in a selected time window. A single oblique slice (close to the coronal plane) is shown, cropped to focus on the kidneys and interpolated to an isotropic voxel size of $2.5 \mathrm{~mm}$. Pyruvate and lactate images are shown interleaved, as acquired, with the first image being of lactate for the fully sampled acquisitions (FS), and the first being of pyruvate for the undersampled acquisitions (US). Within each row, pyruvate and lactate images are scaled separately to their maximum value within the given time window.

Theoretical pre-calibration

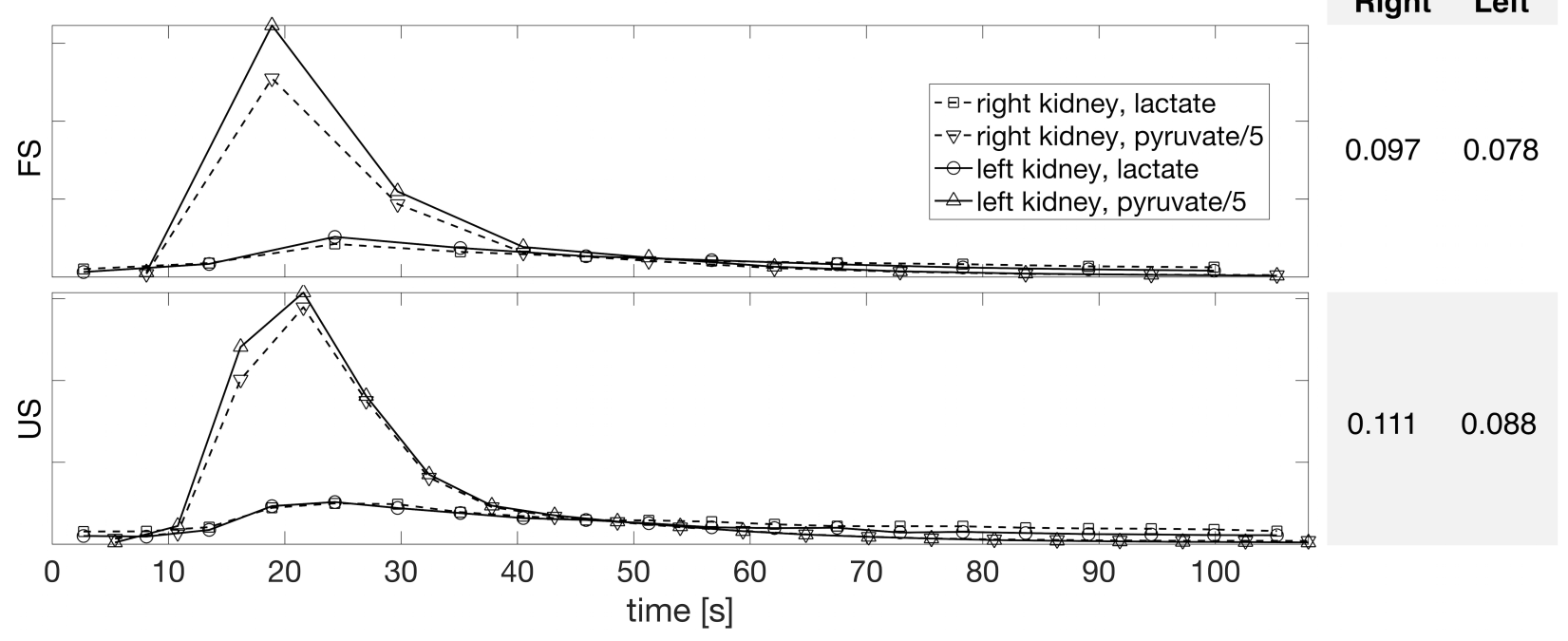

AUC ratio (lactate/pyruvate) Renal cortex

Right Left

Figure 8: Pyruvate and lactate dynamics for the theoretical pre-calibrated reconstruction. The curves represent mean values for the left and right renal cortices, respectively. The table values to the right show area under the curve (AUC) ratios for the lactate and pyruvate curves. 


\section{SUPPORTING INFORMATION}

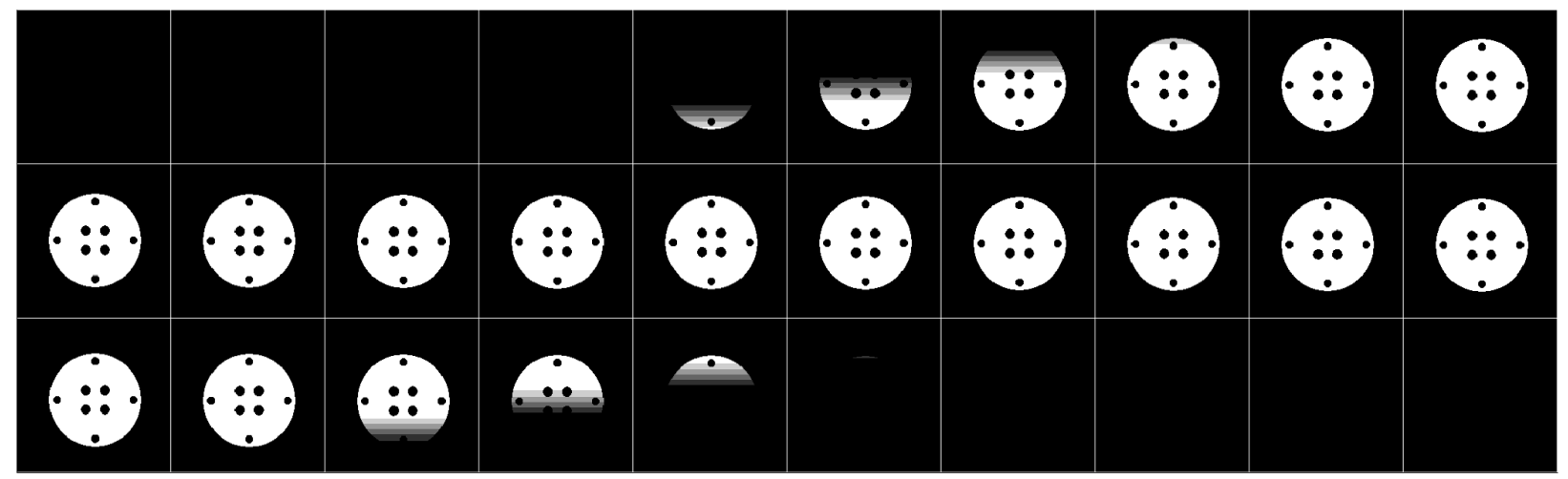

Figure S1: Digital phantom, "true sample", shown in the axial plane with z-resolution and FOV matched to the reconstructed resolution in $z$. 

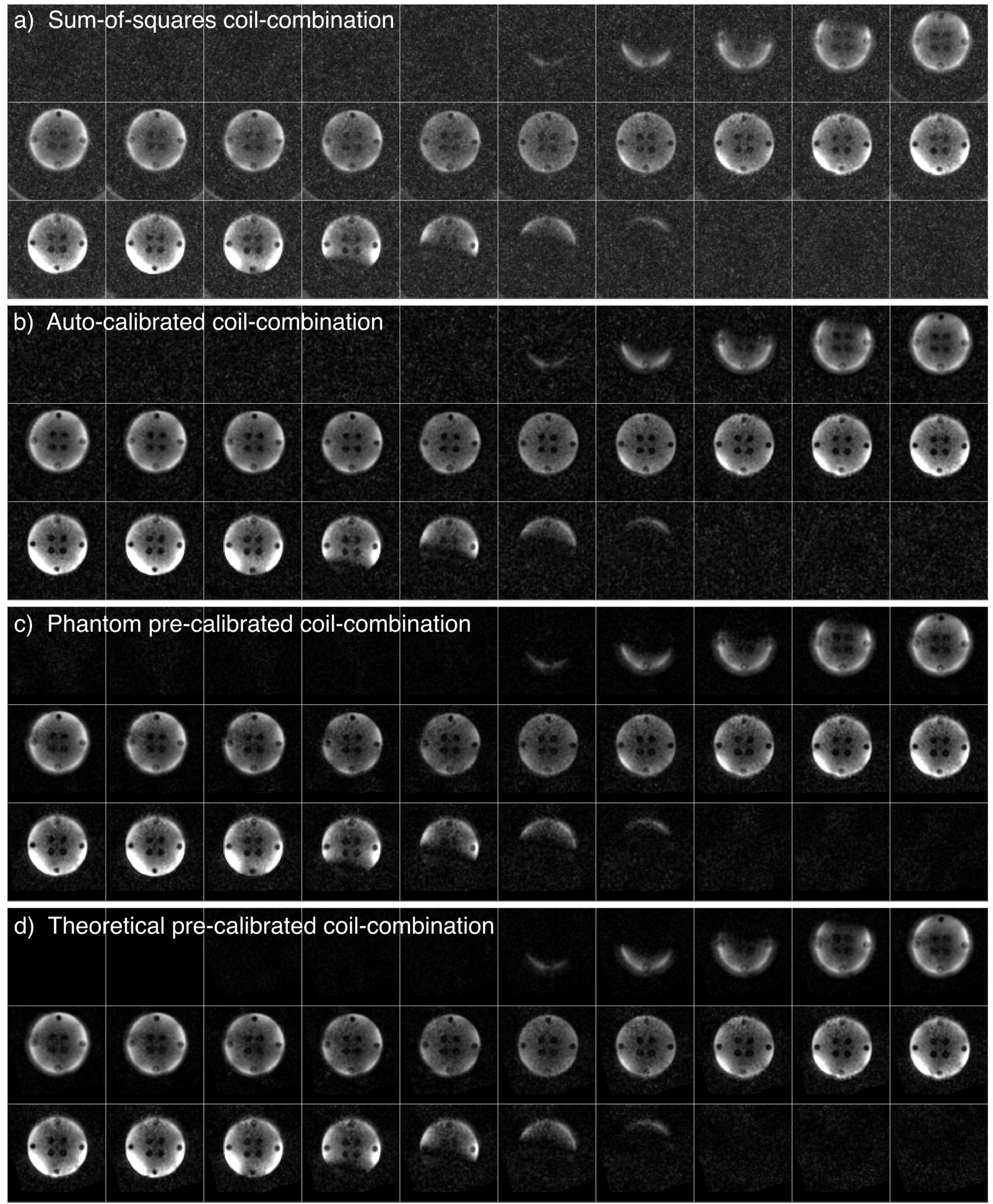

Figure S2: Reconstruction of the fully sampled data with different coil-combination methods: a) sum-ofsquares, b) combination with auto-calibrated sensitivities, c) combination with phantom pre-calibration maps, d) combination with theoretical pre-calibration maps. To make the comparison to sum-of-squares coilcombination fair, these reconstructions did not include off-resonance correction or intensity correction. All images are shown with equal window settings. 
a)
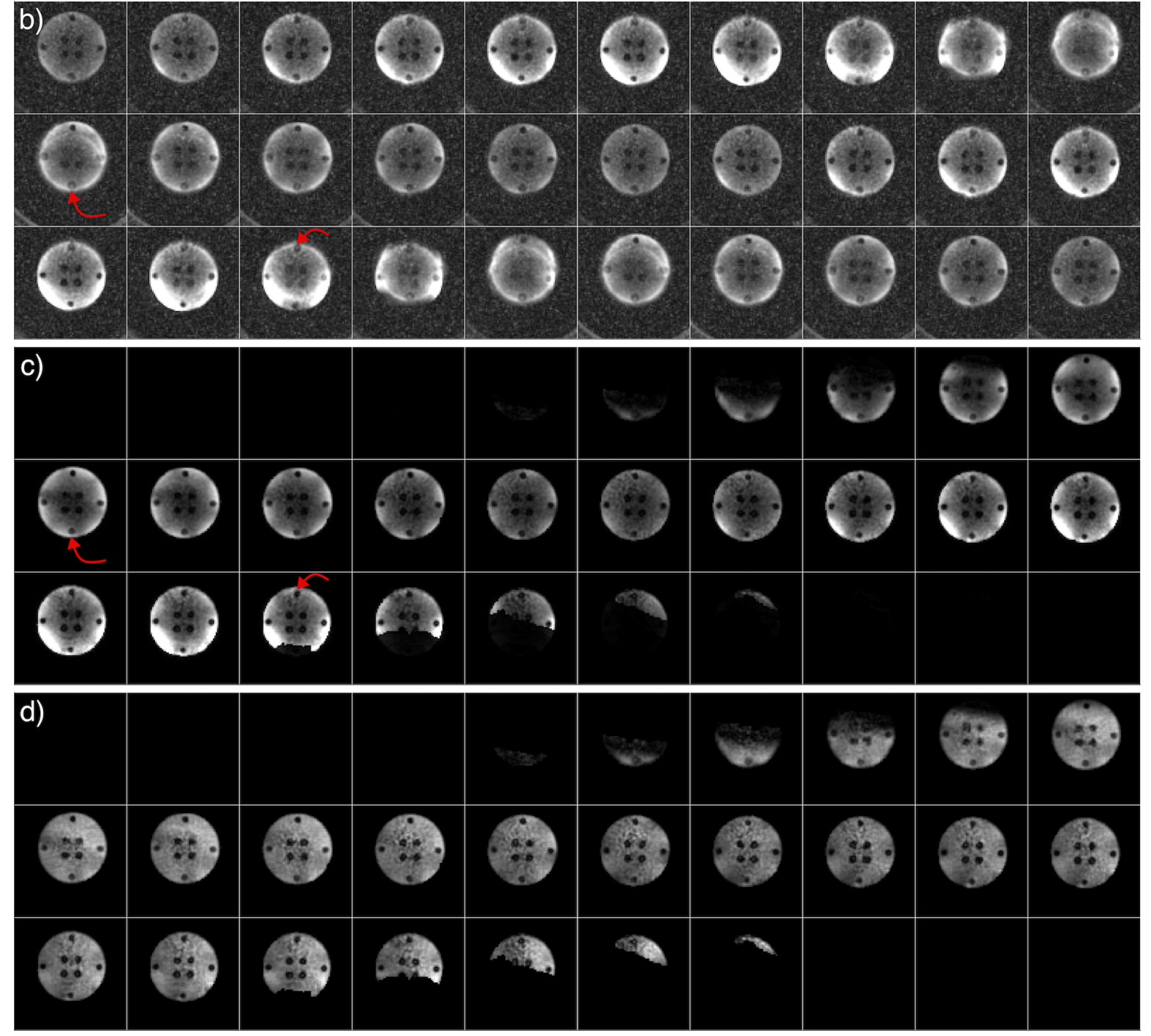

Figure S3: Axial slice representation of the full acquisition volume for the uniformly undersampled data. a) Smoothed and masked $\mathrm{B}_{0}$-field map in units of $\mathrm{Hz}$, scaled and shifted to the ${ }^{13} \mathrm{C}$ frequency. b) Undersampled data after sum-of-squares combination. c) CG-SENSE reconstruction using theoretical sensitivities and inhomogeneity correction. The red arrows point to two regions off-resonance (see a)), which led to distortions in b), but were successfully corrected for in c). d) The reconstruction in c) after intensity correction. 
Table S1: Physiological state of the pig during the scan session.

\begin{tabular}{|c|c|c|c|c|c|c|c|c|c|c|c|c|c|c|}
\hline & $\begin{array}{c}\text { Time } \\
\text { (hh:mm) }\end{array}$ & $\begin{array}{r}\text { tidalV } \\
(\mathrm{mL})\end{array}$ & $\begin{array}{l}\text { resp. F. } \\
(1 / \mathrm{min} .)\end{array}$ & $\begin{array}{l}\mathrm{CO}_{2} \\
(\%)\end{array}$ & $\begin{array}{c}\mathrm{O}_{2} \\
(\%)\end{array}$ & $\begin{array}{c}\text { HF } \\
(1 / \mathrm{min} .)\end{array}$ & $\begin{array}{l}\text { Sat. } \\
(\%)\end{array}$ & $\begin{array}{l}\text { BP sys. } \\
\text { (mmHg) }\end{array}$ & $\begin{array}{l}\text { BP dia. } \\
\text { (mmHg) }\end{array}$ & $\begin{array}{l}\text { BP MAP } \\
(\mathrm{mmHg})\end{array}$ & $\begin{array}{l}\text { temp. } \\
\left({ }^{\circ} \mathrm{C}\right)\end{array}$ & $\mathrm{pH}$ & $\begin{array}{c}\text { cGlu } \\
(\mathrm{mmol} / \mathrm{L})\end{array}$ & $\begin{array}{c}\text { cLac } \\
(\mathrm{mmol} / \mathrm{L})\end{array}$ \\
\hline Baseline & $10: 00$ & 406 & 12 & 5.1 & 60 & 55 & 100 & 119 & 64 & 83 & 36.6 & 7.49 & 3.9 & 1.4 \\
\hline 1st inj. & $12: 20$ & 357 & 14 & 4.7 & 60 & 54 & - & 125 & 69 & 88 & - & 7.48 & 2.0 & 1.0 \\
\hline 2nd inj. & $12: 53$ & 366 & 14 & 4.7 & 60 & 56 & - & 124 & 67 & 85 & - & 7.49 & 2.4 & 1.0 \\
\hline 3rd inj. & $13: 20$ & 364 & 14 & 4.6 & 60 & 57 & - & 128 & 66 & 86 & - & 7.51 & 2.7 & 0.9 \\
\hline
\end{tabular}

